\title{
BJOG-20-2353.R2 What should we believe when systematic reviews disagree?
}

\author{
Andrew Weeks ${ }^{1}$ \\ ${ }^{1}$ University of Liverpool
}

May 17, 2021

\section{BJOG-20-2353.R2 What should we believe when systematic reviews disagree?}

For many years, uterine balloon tamponade (UBT) has been used to treat severe postpartum haemorrhage $(\mathrm{PPH})$, despite a lack of randomised trials to demonstrate its effectiveness. With commercial devices being expensive, clinicians in low resource settings have made their own using 2 low-cost, widely available items (Foley catheters and condoms). Public health experts have been so confident of their benefit that large programmes have been set up to disseminate the necessary skills worldwide.

Recently, however, the global maternal health community has been thrown into disarray when not one, but two randomised controlled efficacy trials suggested that outcomes with condom catheter UBTs were actuallyworse than normal care in low resource settings. The Cochrane meta-analysis concluded that "in [low resource] settings, balloon tamponade [should be] only introduced alongside multi-system improvements in PPH care" (Kellie et al. Cochrane Database of Systematic Reviews 2020(7): CD013663).

Systematic reviews may be the pinnacle of evidence-based medicine, but even they can differ in how to interpret evidence. And so, proponents of the condom catheter conducted their own systematic review with far wider inclusion criteria - they not only included the randomised trials but examined success rates from 15 non-randomised trials and 69 case series (Suarez et al. Am J Obstet Gynecol 2020;222(4):293.e1-e52). Furthermore, the primary outcome was the success rate of the technique (overall 86\%) rather than the risk of morbidity and mortality compared to controls, as used in the Cochrane review. This is problematic, as reported 'success rates' without controls can be very difficult to interpret: in initial case series misoprostol showed similar success rates against life-threatening haemorrhage before RCTs eventually showed it to be less effective than oxytocin.

A third version of the same review is published today by a WHO team (Pingray et al. BJOG 2021;XXX,XXX). This time they include only 4 high quality studies in which UBT was compared to standard care. With a composite maternal morbidity / mortality outcome, they found no evidence of benefit and concluded that "the effect ... is unclear, as is the role of the type of device and the setting". WHO studies are now underway to address this uncertainty.

But why is this all so important? The difficulty comes because the World Health Organisation has been updating its guidance on PPH management, and had to declare a position on UBT. The recently-published guideline, drawn up by independent experts, accepts the validity of the RCTs but recognises that there is wide acceptability of the technique and that the evidence of harm is only for condom catheters and onlyin resource-poor settings. They put a high emphasis on minimising harm and conclude that UBTs should only be used in settings where there is already a good standard of care, including recourse to blood transfusion and surgery if needed (WHO. Geneva: World Health Organization; 2021).

Until further studies are published, the debate will continue. But this episode shows how the choice of inclusion criteria and outcomes in systematic reviews are critical, both for their conclusions, and for global 
policy. 